\title{
COVID-19 in Children: Clinical Approach and Management
}

\author{
Jhuma Sankar $^{1} \cdot$ Nitin Dhochak ${ }^{1} \cdot$ S. K. Kabra ${ }^{1} \cdot$ Rakesh Lodha $^{1}$ \\ Received: 2 April 2020 / Accepted: 2 April 2020 / Published online: 27 April 2020 \\ (C) Dr. K C Chaudhuri Foundation 2020
}

\begin{abstract}
COVID-19 pandemic caused by severe acute respiratory syndrome coronavirus 2 (SARS-CoV-2) is a major public health crisis threatening humanity at this point in time. Transmission of the infection occurs by inhalation of infected droplets or direct contact with soiled surfaces and fomites. It should be suspected in all symptomatic children who have undertaken international travel in the last $14 \mathrm{~d}$, all hospitalized children with severe acute respiratory illness, and asymptomatic direct and high-risk contacts of a confirmed case. Clinical symptoms are similar to any acute respiratory viral infection with less pronounced nasal symptoms. Disease seems to be milder in children, but situation appears to be changing. Infants and young children had relatively more severe illness than older children. The case fatality rate is low in children. Diagnosis can be confirmed by Reverse transcriptase - Polymerase chain reaction (RT-PCR) on respiratory specimen (commonly nasopharyngeal and oropharyngeal swab). Rapid progress is being made to develop rapid diagnostic tests, which will help ramp up the capacity to test and also reduce the time to getting test results. Management is mainly supportive care. In severe pneumonia and critically ill children, trial of hydroxychloroquine or lopinavir/ritonavir should be considered. As per current policy, children with mild disease also need to be hospitalized; if this is not feasible, these children may be managed on ambulatory basis with strict home isolation. Pneumonia, severe disease and critical illness require admission and aggressive management for acute lung injury and shock and/or multiorgan dysfunction, if present. An early intubation is preferred over non-invasive ventilation or heated, humidified, high flow nasal cannula oxygen, as these may generate aerosols increasing the risk of infection in health care personnel. To prevent post discharge dissemination of infection, home isolation for 1-2 wk may be advised. As of now, no vaccine or specific chemotherapeutic agents are approved for children.
\end{abstract}

Keywords Acute lung injury - Acute respiratory distress syndrome - ARDS · COVID-19 • Multiorgan dysfunction · Hydroxychloroquine $\cdot$ Lopinavir $\cdot$ Ritonavir $\cdot$ SARS-CoV-2 $\cdot$ Severe pneumonia

\section{Background}

Coronavirus disease 2019 (COVID-19) pandemic caused by the severe acute respiratory syndrome coronavirus 2 (SARS$\mathrm{CoV}-2$ ) is a major public health crisis threatening humanity at this point in time. The virus possibly originated in bats and was transmitted to humans through yet unknown intermediary animals in Wuhan, Hubei province, China in December 2019 $[1,2]$. As per the WHO estimates of 16th April 2020, there are 1,991,562 cases, and 130,885 deaths [3]. In India, the reported cases as on 16th April were 12949 cases and 437 deaths [4].

Rakesh Lodha

rakesh_lodha@hotmail.com; rlodha1661@gmail.com

1 Department of Pediatrics, All India Institute of Medical Sciences, New Delhi 110029, India
Modes of transmission of disease are direct inhalation of infected droplets (produced during coughing or sneezing by infected person) and direct contact with surfaces and fomites soiled by infected respiratory secretions. Special situations associated with aerosol generation (cardiopulmonary resuscitation, endotracheal intubation, open suctioning, bronchoscopy, nebulisation and use of non-invasive ventilation) can lead to aerosol generation and spread. Virus has also been isolated from stool samples but contribution of infection spread by the feco-oral route is not established [5]. Exact duration of viability of virus on surfaces may be few hours to few days; the survival duration is likely to be considerably low at higher temperature and low humidity conditions [6].

Median (IQR) incubation period has been assessed to be $5.1(4.5,5.8) \mathrm{d}$ while 101 in 10,000 patients may develop symptoms after $14 \mathrm{~d}$ [7]. The clinical symptoms are similar to that of any other acute respiratory viral infection and include fever, cough, sore throat, breathlessness, fatigue, 
malaise, etc. It has been observed that nasal symptoms are less pronounced in SARS-CoV-2 infection. The disease is mild in about $80 \%$ of the reported cases. In those with co-morbidities and elderly, the disease is often severe progressing to acute respiratory distress syndrome (ARDS) and multi organ dysfunction [8]. The case fatality rate is estimated to range from 0.25 to $3 \%$; it is much higher in elderly and those with comorbidities [9]. Based on the current data, children seem to be less frequently affected and the disease may be less severe; however, the situation may change. Children however may have asymptomatic infection and also prolonged fecal-shedding, which may contribute to the spread of the pandemic [10].

Diagnosis is confirmed by demonstration of the virus in respiratory specimen (nasopharyngeal and oropharyngeal swab/ endotracheal secretions or bronchoalveolar lavage) by using specific RT-PCR. Recently, point of care antibody tests have also been approved; their role in clinical management needs to be defined. Common laboratory findings include normal/ low white cell counts with elevated C-reactive protein (CRP). X-ray and CT scan pictures in moderate to severe illness are suggestive of diffuse bilateral involvement of lungs [11]. Treatment consists of mainly supportive care; while role of antiviral agents is being evaluated. Prevention is the key and home isolation of exposed individuals, management of symptomatic cases in healthcare facilities, and strict infection control measures at hospitals may help break the chain of transmission [12].

\section{Definitions}

The criteria for suspecting SARS-CoV-2 infection are being periodically updated by the Indian Council of Medical Research (ICMR) and the Government of India [13].

\section{When to Suspect in Children}

1. All symptomatic children who have undertaken international travel in the last $14 \mathrm{~d}$

OR

2. All hospitalized children with severe acute respiratory illness (fever and cough and/or shortness of breath)

OR

3. Asymptomatic direct and high-risk contacts of a confirmed case (should be tested once between day 5 and day 14 after contact)
Symptomatic refers to children with any symptoms of fever, cough, or shortness of breath. Direct and high-risk contacts include those who live in the same household with a confirmed case or area (designated hot spot) and health care professional (HCP) who examined a confirmed case.

These definitions will have to be modified if there is convincing evidence of community transmission.

\section{Confirmed Case}

A person with laboratory confirmation of SARS-CoV-2 infection, irrespective of clinical signs and symptoms.

\section{Clinical Features in Children}

Initial three pediatric case series reported 171,20 , and 10 children, respectively from Wuhan and other provinces of China $[10,14,15]$. After incubation period of $2-14 \mathrm{~d}$, children become symptomatic. In the case series of 171 children with RT-PCR confirmed SARS-CoV-2 infection, signs and symptoms included cough (48.5\%), pharyngeal erythema (46.2\%), fever $(41.5 \%)$, fast breathing $(28.7 \%)$, diarrhea $(8.8 \%)$, rhinorrhea $(7.6 \%)$, fatigue $(7.6 \%)$, and vomiting $(6.4 \%)$. Frequency of asymptomatic infection, upper respiratory tract infection and pneumonia was $15.8 \%, 19.3 \%$ and $64.9 \%$, respectively in this study. One child died [15].

The largest pediatric series, till date, of 2143 patients reported $34 \%$ laboratory confirmed cases and $66 \%$ suspected cases [16]. The median (IQR) age was $7(2,13)$ y and 1213 cases $(57 \%)$ were boys. Among laboratory confirmed cases, proportion of asymptomatic, mild, moderate, severe and critically ill infections was $12.9 \%, 43.1 \%, 41 \%, 2.5 \%$ and $0.4 \%$, respectively. Children with confirmed diagnosis had milder clinical course than suspected cases (which could include other prevalent viruses like respiratory syncytial virus, influenza, and para-influenza etc. in this age group); indicating that severity of SARS-CoV-2 illness could be milder than other acute respiratory infections in this age group. The median time from onset of illness to diagnosis was $2 \mathrm{~d}$ (range: 0-42 d). Proportion of 'severe and critical' cases was $10.6 \%, 7.3 \%$, $4.2 \%, 4.1 \%$ and $3.0 \%$ for the age group of $<1,1-5,6-10$, $11-15$ and $>15 \mathrm{y}$, respectively; indicating that young children, especially infants, were more vulnerable to severe SARS$\mathrm{CoV}-2$ infection. One child (14-y-old) died. This study didn't describe frequency of individual symptoms in their population [16]. Patients with severe manifestation usually develop features of hypoxemia and hypoperfusion by end of first week. Commonly described complications are acute respiratory distress syndrome (ARDS), myocarditis, septic shock, disseminated intravascular coagulation, acute kidney injury and liver dysfunction [17]. Children usually have milder disease. Estimated mortality in analysis of Chinese patients in age 
group $0-9$ y and $10-19$ y was $0 \%$ and $0.18 \%$, respectively compared to overall mortality of $2.3 \%$, and $8 \%$ in age group $70-79$ y [18].

\section{Laboratory Diagnosis}

As per directive from Ministry of Health and Family Welfare (MoHFW), Government of India, all suspected cases are to be reported to district and state surveillance officers. The helpline number is 011-23978046.

Preferred diagnostic test is Reverse transcriptase Polymerase chain reaction (RT-PCR) for SARS-CoV-2 RNA. Various commercially available kits are approved by ICMR for use in India [19]. Next generation sequencing is utilized to identify SARS-CoV-2 strains and mutations for epidemiological and research purposes. Rapid serology kits may not be positive during first 7-10 d of infection and stay positive for several weeks following infection. These kits have been approved for clinical use recently [20].

Preferred sample for children not receiving mechanical ventilation are upper respiratory tract sample (nasopharyngeal and oropharyngeal swab); these should be transported in viral transport media (VTM) on ice. In mechanically ventilated children, bronchoalveolar lavage (BAL) or endotracheal aspirate would be the preferred specimen. These have to be mixed with the viral transport medium and transported on ice. Sputum induction should be avoided in view of risk of aerosol generation [21]. In a study of RT-PCR confirmed patients, the sensitivity for different specimens was as follows: BAL (14/15; 93\%), sputum $(75 / 104 ; 72 \%)$, nasal swab $(5 / 8 ; 63 \%)$, bronchoscopic brush biopsy $(6 / 13 ; 46 \%)$, pharyngeal swab (126/398; 32\%), feces $(44 / 153 ; 29 \%)$, blood $(3 / 307 ; 1 \%)$, and urine $(0 / 72)$ [5]. In a symptomatic child, the viral load is expected to be high and single test using upper respiratory tract sample (nasopharyngeal and oropharyngeal swab) should be able to detect almost all the cases.

\section{General Guidelines}

- $\quad$ Samples will be tested for SARS-CoV-2 at laboratories (government or private) approved by the Government of India

- Trained health care professionals to wear appropriate personal protective equipment (PPE) with latex free purple nitrile gloves while collecting the sample from the patient. Proper infection control precautions should be maintained when collecting specimens.

- Entry of visitors or attendants during sample collection is to be restricted.

- Requisition form for each specimen should be completed and submitted.

- Proper disposal of all waste generated has to be ensured (red container).
Respiratory Specimen Collection Methods

\section{Upper Respiratory Tract}

Nasopharyngeal and Oropharyngeal (Throat) Swab [21]

1. Use only synthetic fiber swabs (e.g., dacron or rayon) with plastic shafts. Do not use cotton or calcium alginate swabs or swabs with wooden shafts.

2. For nasopharyngeal swab, tilt patient's head back 70 degrees. Insert the swab in one nostril and push deep along floor and septum of nose until resistance is felt. Rotate the swab several times against nasopharyngeal wall. Place tip of the swab into sterile viral transport media tube and cut off the applicator stick.

3. For throat swab, take a second dry swab, insert into mouth, and swab the posterior pharynx. Avoid touching the tongue, teeth, gums and tonsils. Place tip of swab into the same tube and cut off the applicator tip.

\section{Lower Respiratory Tract}

BAL and Endotracheal Aspirate Collect 2-3 ml into a sterile, leak-proof, screw-cap sputum collection cup or sterile dry container (mucous trap).

\section{Infection Control}

Infection prevention and control are important aspects of the care; appropriate guidelines should be followed [22].

Patients suspected of having SARS-CoV-2 infection should be shifted to the isolation facility/ designated COVID-19 areas from the triage area as soon as possible. The HCP should be handling the patients after donning appropriate PPE according to their level of exposure as described [22].

\section{Management}

Figure 1 shows the clinical decision pathway for patients presenting with acute respiratory illness in out-patient department (OPD)/ emergency room (ER).

At present all children presenting with severe acute respiratory illness (SARI) are to be subjected to RT-PCR for SARS-CoV-2. This is based on the guideline issued by ICMR. However, due to high background rate of acute respiratory tract infections due to other etiologies, acute bronchiolitis, acute severe asthma, etc. in children, a large number of them may fulfil case definition of SARI and may get admitted in a common facility along with adults suspected to have COVID-19. The latter are more likely to have COVID-19. This may predispose children who are less likely to be 


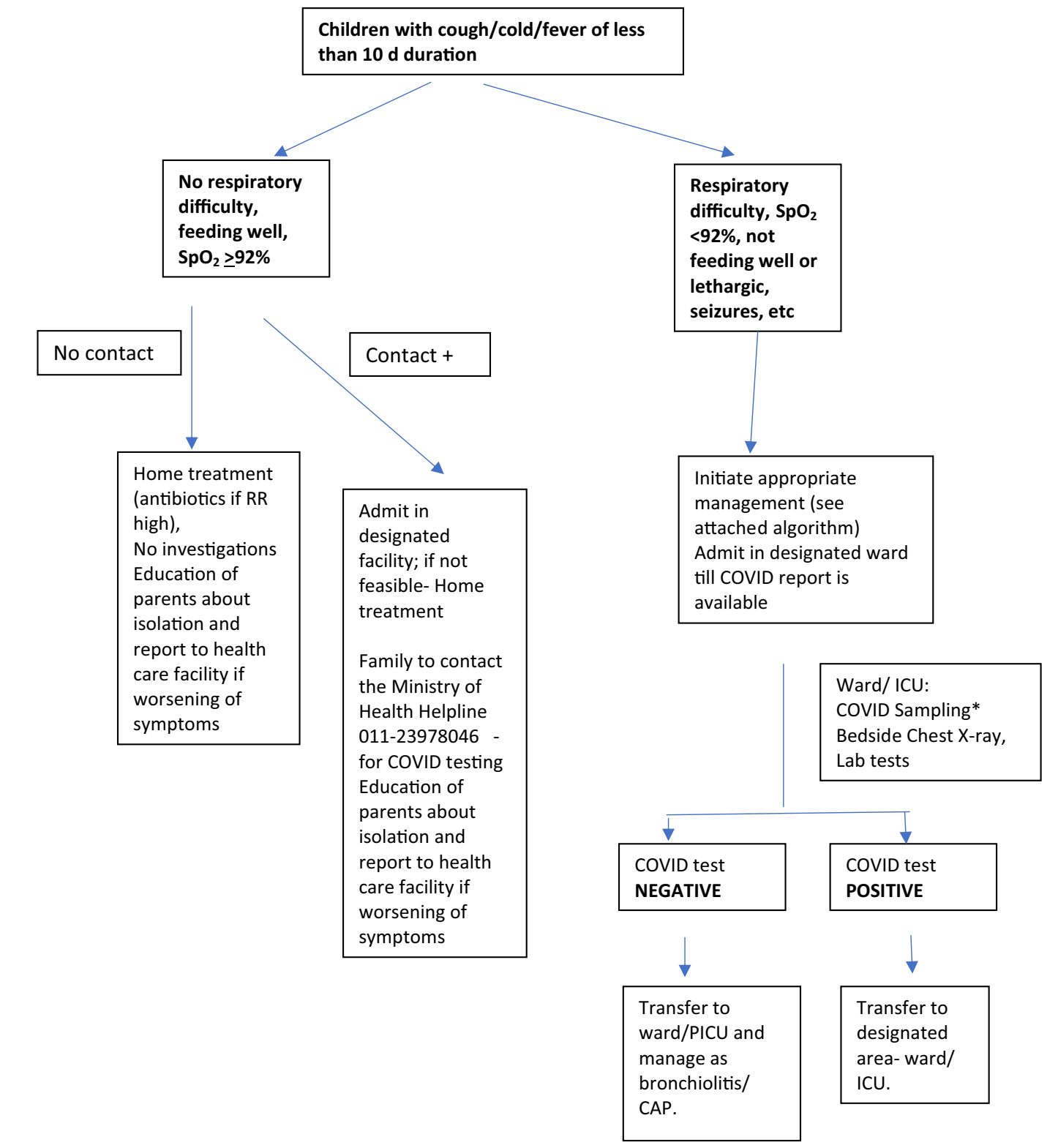

\section{*Testing}

Respiratory sample: Nasopharyngeal and oropharyngeal swab together sent in viral transport medium, posterior-pharyngeal swab, endotracheal aspirate, or bronchoalveolar lavage.

Fig. 1 Approach to a child with acute respiratory symptoms during COVID-19 pandemic

SARS-CoV-2 infected to get exposed to the novel virus; it may be appropriate to have a separate cubicle for children suspected to have COVID-19. There is a need to have an alternative case definition/ indication for getting them tested. Till alternative definitions are developed, children suspected to have SARS-CoV-2 infection awaiting results should be admitted separate from adults. Additionally, parents will be required to stay with children isolated because of suspected corona infection; they need to be provided appropriate PPE. A rapid turn-around time for the test reports will also reduce the risk of exposure to a minimum.
Currently, children with mild illness without a history of contact are not subjected to test for SARS-CoV-2 [13]. In the coming weeks, it may change and even it may be carried out in mild illness also.

\section{Indications for Hospital Admission}

At present, the official guidelines recommend admission for all confirmed cases. Particularly, patients classified as "Severe Pneumonia" and "Critically ill" should be admitted to hospital for further care (Fig. 2). The following criteria may 


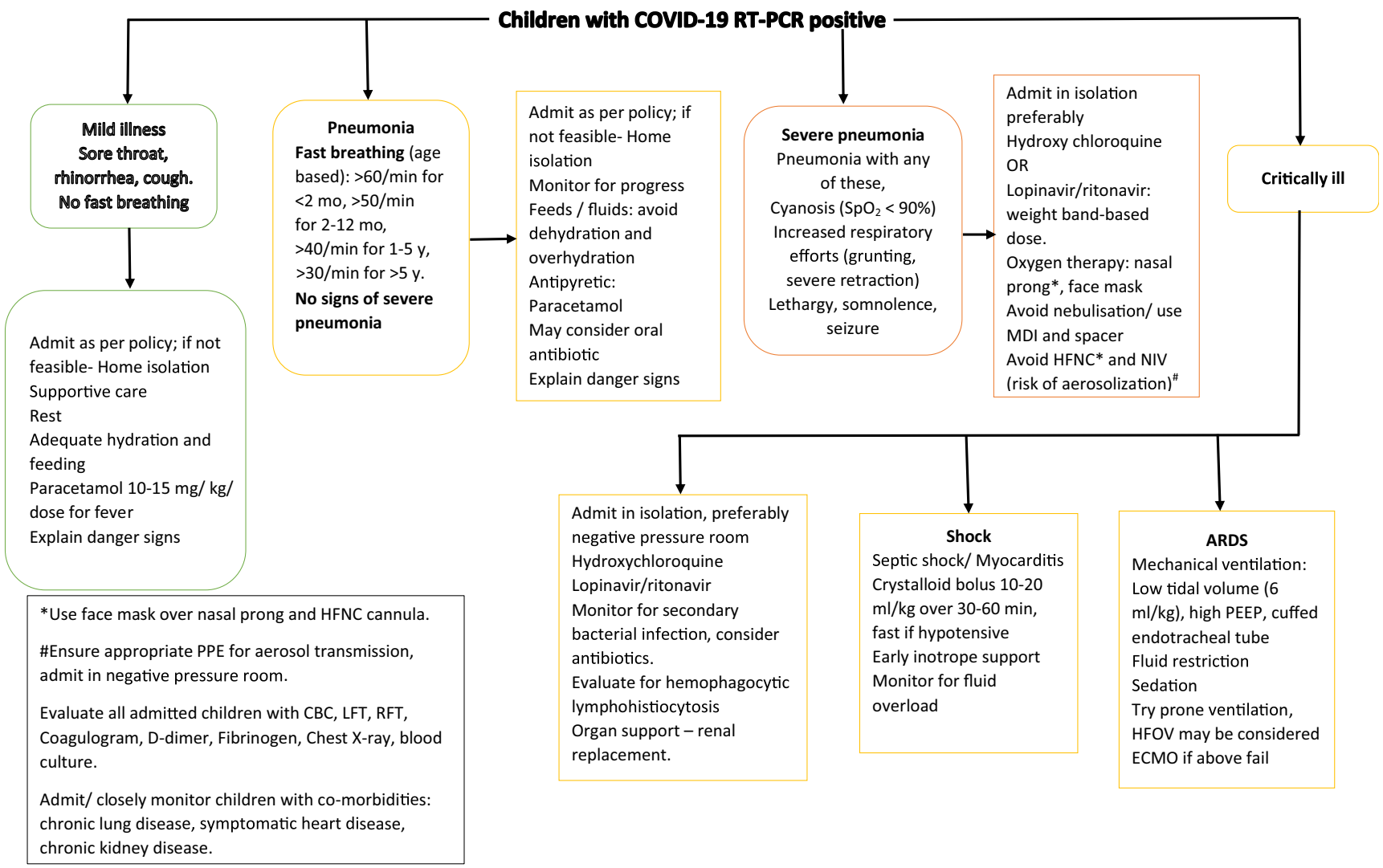

Fig. 2 Summary of management of children with COVID-19

particularly be considered for admission (Any ONE of the following criteria) [21]:

1. Respiratory distress

2. $\mathrm{SpO}_{2}<92 \%$ on room air

3. Shock/ poor peripheral perfusion

4. Poor oral intake, especially in infants and young children

5. Lethargic, especially in infants and young children

6. Seizures/ encephalopathy

At present there is no separate guideline for children who have underlying illness including chronic respiratory illnesses, immunocompromised states, uncorrected heart disease, chronic renal disease etc. This population needs more intensive monitoring and early therapy [13].

\section{Investigations in Admitted Patients}

Chest Imaging Chest X-ray (CXR) is usually the first-line investigation due to ease of availability. CXR findings are not described in detail in pediatric studies. Adult series of COVID19 showed consolidation (47\%) and ground glass opacities (GGO) (33\%) as commonest parenchymal lesions with more peripheral $(41 \%)$ and lower zone (50\%) and frequently bilateral $(50 \%)$ distribution [23]. In pediatric series, common patterns on CT scan showed GGO (32.7\%), local patchy shadow (18.7\%), bilateral patchy shadow (12.3\%), and interstitial abnormalities (1.2\%) [15]. Another study of CT scan in children with COVID19 , showed more bilateral $(50 \%)$ than unilateral involvement (30\%). Common patterns of CT involvement were GGO, consolidation with surrounding halo sign, fresh mesh shadow and tiny nodules [14]. These findings represent interstitial involvement of parenchyma. Chest X-rays should be considered in children requiring oxygen at admission or showing increase in respiratory distress or increase in requirement of respiratory support suggesting disease progression. This may suggest severe illness or early deterioration. Children not admitted to HDU or ICU may require a chest X-ray if they have worsening hypoxemia, particularly, if they have pre-existing conditions.

Other Investigations are needed in admitted patients. Complete blood counts is commonly performed. Extent of leukopenia, and lymphopenia is lesser in children with COVID-19 than adults $[8,15]$. Median (IQR) of total leukocyte counts, lymphocytes and neutrophil counts in pediatric series were $6800(5500,8200)$ per cu mm, $2900(2200,4400)$ per cu mm, and $2500(1800,3700)$ per cu mm, respectively [15]. Serum chemistry (liver and kidney function test), coagulogram and arterial blood gases, should be frequently monitored in sick children. Procalcitonin (64\%) is more frequently elevated in children than C-reactive protein (19.7\%) [15]. A bedside ultrasound of chest may be done if expertise is available. 


\section{Treatment}

Approach to a child confirmed to be COVID-19 by RT-PCR or a suspected case in whom SARS-CoV-2 virus test is inconclusive or a severely ill patient whose RT-PCR results are awaited, is demonstrated in Fig. 2.

As per the protocol the management would be as follows.

\section{Mild Illness: These Children Have No Respiratory Difficulty, Are Feeding Well, Have $\mathrm{SpO}_{2}>92 \%$}

1. The current guidelines recommend admission in a isolation facility for all positive cases. If this is not feasible, then the child would have to be isolated at home and managed; in this scenario, teleconsultations may have a role.

2. Appropriate antibiotic may be prescribed, if respiratory rate is high.

3. Supportive care: Control of fever using paracetamol (10$15 \mathrm{mg} / \mathrm{kg} /$ dose SOS/ q 4-6 hourly if required); avoid ibuprofen and other NSAIDs.

4. Ensure adequate hydration

5. Danger signs should be explained

6. The parent/ caregiver should take the necessary precautions, use appropriate PPE including a mask.

Duration of Isolation Afebrile for $72 \mathrm{~h}$ AND at least $7 \mathrm{~d}$ after symptom resolution $O R 2$ negative samples $24 \mathrm{~h}$ apart.

\section{Management of Hospitalized Cases}

\section{General Measures}

1. Oxygen supplementation to maintain $\mathrm{SpO}_{2}>92 \%$.

2. Conservative fluid management is followed in mechanically ventilated patients (restrict fluid to $70-80 \%$ maintenance, if there is no evidence of hypovolemia).

3. Symptomatic treatment: Paracetamol for fever ((10$15 \mathrm{mg} / \mathrm{kg} /$ dose SOS/ q 4-6 hourly if required); avoid ibuprofen and other NSAIDs

4. Blood culture sample should be sent at time of admission before starting anti-microbials.

5. Empirical antimicrobials (e.g., Ceftriaxone) within $1 \mathrm{~h}$ of admission in case of suspected sepsis and septic shock.

6. Oseltamivir may be considered after sending appropriate investigation if influenza is suspected.

7. Systemic corticosteroids are not recommended, unless indicated for any other reason.

8. MDI with spacer is preferred for administration of inhaled medication over nebulization, as nebulization is associated with increased risk of aerosolization [24].
9. Close monitoring for worsening clinical status is of paramount importance. Children who have significant distress may be managed in a HDU setting; those needing intubation and mechanical ventilation or other organ support should be managed in an ICU.

Respiratory Support Low flow oxygen cannula is utilized with flows up to $1-2 \mathrm{~L} / \mathrm{min}$ in infants, $2-4 \mathrm{~L} / \mathrm{min}$ in young children and 4-6 L/min in older children and adolescents. Heated humidified high flow nasal cannula (HHHFNC) is not favoured as there are concerns of an increased aerosol formation. Similarly use of NIV is discouraged in view of potential for aerosol generation, though clinical evidence is not definitive [24]. If HHHFNC or NIV are used, HCP should wear personal protective equipment (PPE) for aerosol transmission, and separate negative pressure isolation room should be provided to the child. A medical mask should be secured on face of the child receiving oxygen therapy with nasal prong or HHHFNC, if the child tolerates. Child should be monitored frequently including for $\mathrm{SpO}_{2}$, change in respiratory rate and heart rate, hemodynamic parameters, sensorium and urine output. Respiratory support should be promptly hiked to mechanical ventilation if there is no benefit of NIV trial on respiratory rate, heart rate and respiratory efforts, or respiratory status worsens.

Specific Therapy No specific antivirals have been proven to be effective as per currently available data. Hydroxychloroquine and chloroquine have been demonstrated to have anti-SARSCoV-2 activity in in-vitro studies. Mechanisms for anti-viral activity of hydroxychloroquine include inhibiting membrane fusion by increasing $\mathrm{pH}$ of endosome/lysosome, inhibiting virus entry by changing glycosylation of ACE2 receptor and spike protein, and immune-modulation [25]. In an openlabelled uncontrolled trial in adults, there was significant reduction in viral load with hydroxychloroquine and azithromycin therapy compared to routine care [26]. Clinical trial of Lopinavir/Ritonavir (protease inhibitors impairing virus assembly) in adults was not associated with improved 28-d mortality ( $19.2 \%$ vs. $25.0 \%$; difference, -5.8 percentage points; $95 \%$ CI, -17.3 to 5.7 ) [27]. But the study only included the most severe patients [27]. Starting the therapy early, before multi-organ dysfunction sets in, might be more appropriate approach. It is to be seen if early therapy with these drugs would have any impact on disease progression and need for mechanical ventilation. Currently there are no pediatric studies. Clinical indications for starting virus-suppressive therapy (either Hydroxychloroquine or Lopinavir/Ritonavir) include patients with suspected or confirmed COVID-19 with "severe pneumonia" or "critically ill patients".

1. Hydroxychloroquine $7-8 \mathrm{mg} / \mathrm{kg} / \mathrm{dose}$ twice daily for Day 1 and then Day 2-5, 7-8 $\mathrm{mg} / \mathrm{kg}$ once a day. 
2. Lopinavir/Ritonavir: Suggested dose of LPV/r is $10 \mathrm{mg} /$ $2.5 \mathrm{mg}$ per $\mathrm{kg}$ twice daily for maximum $14 \mathrm{~d}$; maximum dose is $400 \mathrm{mg} / 100 \mathrm{mg}$ twice daily. Choose appropriate formulation to deliver the calculated dose (Syp LPV/r $80 \mathrm{mg} / 20 \mathrm{mg}$ per ml; Tablet LPV/r $100 \mathrm{mg} / 25 \mathrm{mg}$; Tablet LPV/r 200 mg/50 mg).

These recommendations are based on a recent guidance issued by ICMR for adults. As the dosing studies are not yet available, the doses mentioned above have been extrapolated from the doses recommended for adults. HCPs should be aware about common side effects of hydroxychloroquine including headache, dizziness, ringing sensation in ears; nausea, vomiting, abdominal pain; loss of appetite, weight loss; mood changes, feeling nervous or irritable; skin rash or itching; low heart rate, hypoglycemia, etc. Lopinavir/ritonavir use can be associated with skin rash, gastrointestinal symptoms, hepatitis, metabolic derangements (hypercholesterolemia, hyperglycemia), neutropenia, thrombocytopenia, and prolonged QT.

Caution Do NOT co-administer Lopinavir/ritonavir and Hydroxychloroquine due to drug interaction which may cause increased Hydroxychloroquine levels and subsequent toxicity (e.g., QT prolongation, hypoglycemia).

As the pandemic is evolving, research is ongoing for more effective therapy for seriously ill patients. Remdesivir (prodrug of remdesivir triphosphate, an adenosine analogue) has been shown as a potent inhibitor of SARS-CoV-2 in-vitro and is undergoing clinical trial in adults with COVID-19 [28]. In critically ill patient with hyper-inflammatory response, anti-IL-6 receptor antibodies (Tocilizumab, Sarilumab) are another promising therapies under investigation [29]. Recently, a case series of 5 adults with ARDS with high viral load were treated with convalescent plasma transfusion (SARS-CoV-2 specific IgG titer greater than 1:1000) with favourable clinical outcome [30]. Other drugs described for SARS-CoV-2 without proven efficacy include antiviral (interferons, ribavirin) and anti-inflammatory (azithromycin) agents $[28,29]$. In treatment refractory patients, therapy can be individualized with one of these agents on case-to-case basis. With ongoing research in therapeutics against SARS-CoV-2, treatment algorithms are likely to evolve drastically.

\section{Criteria for ICU Admission}

- Requiring mechanical ventilation

- Shock requiring vasopressor support

- Worsening mental status

- Multi-organ dysfunction syndrome

\section{Indications for Intubation}

- Severe respiratory distress; exhaustion.
- Not able to maintain $\mathrm{SpO}_{2}>90 \%$ on non-invasive oxygen supplementation

- $\mathrm{PaO}_{2} / \mathrm{FiO}_{2}<200$.

- $\mathrm{PaO}_{2} / \mathrm{FiO}_{2}<300$ with hypotension requiring vasopressor support

- $\mathrm{GCS}<8$ with threatened airway

- Decision to intubate should be taken on a case by case basis based on the clinician's discretion

\section{How to Intubate}

- Pre-oxygenation with $100 \% \mathrm{FiO}_{2}$ with non-rebreathing mask or nasal prongs.

- Try to avoid bag and mask ventilation (risk of aerosol generation). If needed, can be used by connecting a viral filter.

- The most skilled member of the team should be identified at the beginning of each shift for performing intubations.

- If readily available, intubation should be performed using a video-laryngoscope.

- Cuffed endotracheal tubes should be used to avoid peritubal leak and dissemination of secretions.

- Rapid sequence intubation should be done.

- During induction, monitor for hemodynamic instability and use fluids and vasopressors, if required.

- Get X-ray chest to confirm correct position of tube.

- After intubation, appropriate cleaning/disinfection of equipment and environment should be done

\section{Management Strategies for ARDS}

The general principles of management of child with ARDS apply to a child with COVID-19 related ARDS. The principles include lung protective ventilation: appropriate high PEEP; and low tidal volume (4-6 ml/kg) [31].

Children with refractory hypoxemia may benefit from ventilation in prone position. For more details, readers may refer to management protocols [32].

\section{Care of Ventilated Patient}

- Fresh, preferably disposable ventilator circuit to be used for every new patient.

- Use viral filter in expiratory limb of the circuit

- Heat and moisture exchanger (HME) to be changed every $48 \mathrm{~h}$ or when visibly soiled

- Use closed suctioning technique and avoid routine suctioning Appropriate sedation should be ensured and intermittent muscle relaxants may be used. 


\section{Special Considerations during Resuscitation}

- Chest compression and bag and mask ventilation should be started only after wearing PPE for aerosol transmission protection.

- Minimize the number of people inside the room during high aerosol generating events like cardiopulmonary resuscitation.

- One airway specialist, one nurse/doctor for chest compression and one nurse for administering medications are essential.

- Other assistants may remain outside the room and may enter only if necessary, after donning full PPE.

- Hand bagging needs to be avoided; if essential use a viral filter with the bag.

\section{Septic Shock}

- Recognize septic shock in children with any hypotension [systolic blood pressure $(\mathrm{SBP})<5$ th centile or $<2 \mathrm{SD}$ below normal for age] or two or more of the following: altered mental state; bradycardia or tachycardia (Heart rate $<90 /$ min or $>160 /$ min in infants, and $<70 /$ min or $>$ $150 /$ min in children); prolonged capillary refill $(>2 \mathrm{~s}$ ) or feeble pulses; tachypnea; mottled or cold skin or petechial or purpuric rash; increased lactate; oliguria; hyperthermia or hypothermia.

- Management should be as per Surviving Sepsis Campaign Guidelines [33]. For more details, refer to management protocols [32].

\section{Supportive Treatment in Critically III Children}

- Head end elevation; avoid if child has poor perfusion/ shock

- Oral hygiene with antiseptic mouthwash

- Glycemic control to maintain blood glucose in range of $100-180 \mathrm{mg} / \mathrm{dl}$

- Foley's catheter for accurate urine output monitoring

- Ryle's tube for enteral feeds/medications

- Central venous catheter

- Bedsore prevention by position change every $2 \mathrm{~h}$

For more details, refer to management protocols [32].

\section{Discharge Criteria}

On resolution of symptoms

- Suspected case - If the laboratory results for SARS-CoV-2 are negative, discharge is to be decided as per discretion of the treating physician based on his provisional/confirmed diagnosis.

- Confirmed case- Resolution of symptoms, radiological improvement with a documented virological clearance in 2 samples at least $24 \mathrm{~h}$ apart.

\section{Instructions for SARS-CoV-2 Positive Mothers who are Breastfeeding their Infants}

A SARS-CoV-2 positive mother who is breastfeeding her infant should continue breastfeeding the infant if her medical condition permits; she should use a medical face mask secured appropriately.

\section{Advice for Parents/ Adults who have COVID-19 and are Staying at Home with a Child}

- The affected person should stay in a separate room.

- The affected person should use a 3-ply surgical mask.

- Household members should stay in a different room and be separated from the person as much as possible.

- Only an assigned family member should be tasked with taking care of the person and should help with groceries, prescriptions and other personal needs.

- Avoid shaking the soiled linen or direct contact with skin.

- Use disposable gloves when cleaning the surfaces or handling soiled linen.

- Wash hands after removing gloves and before and after eating, drinking and using the washroom with soap and water (at least $20 \mathrm{~s}$ ) or with alcohol-based hand sanitizer (at least $30 \mathrm{~s}$ ).

- Toys that the child plays with should be washed frequently, wherever possible.

- Currently, no prophylaxis is recommended for children. They should not be administered hydroxychloroquine.

\section{Environmental Sanitation}

- Immediately remove and wash clothes and bedding that have blood, stool or other body fluids on them.

- Clean and disinfect frequently touched surfaces in the quarantined person's room (e.g., bed frames, tables etc.) daily with Sodium Hypochlorite solution (1\%) or ordinary bleach $(5 \%)$.

- Clean and disinfect toilet surfaces daily with regular household bleach solution/phenolic disinfectants. 
- Wash laundry used by the person separately using common household detergent and dry thoroughly using the warmest temperatures recommended on the clothing label.

- Place all used disposable gloves, masks and other contaminated waste in a lined container before disposing them with other household waste and wash hands with soap and water/alcohol-based hand rub.

Authors' Contributions JS, ND, SKK, RL performed the review of literature and wrote the article. RL will act as guarantor for this paper.

\section{Compliance with Ethical Standards}

Conflict of Interest None.

\section{References}

1. Singhal T. A review of coronavirus disease-2019 (COVID-19). Indian J Pediatr. 2020;87:281-6.

2. Zhu N, Zhang D, Wang W, et al. A novel coronavirus from patients with pneumonia in China, 2019. N Engl J Med. 2020;382:727-33.

3. Coronavirus disease (COVID-19) outbreak situation. Available at: https://www.who.int/emergencies/diseases/novel-coronavirus2019. Accessed 17 April 2020.

4. COVID-19. Available at: https://mygov.in/covid-19/. Accessed 17 April 2020.

5. Wang $\mathrm{W}, \mathrm{Xu} \mathrm{Y}, \mathrm{Gao} \mathrm{R}$, et al. Detection of SARS-CoV-2 in different types of clinical specimens. JAMA. 2020. https://doi.org/10.1001/ jama.2020.3786.

6. van Doremalen N, Bushmaker T, Morris DH, et al. Aerosol and surface stability of SARS-CoV-2 as compared with SARS-CoV-1. N Engl J Med. 2020. https://doi.org/10.1056/NEJMc2004973.

7. Lauer SA, Grantz KH, Bi Q, et al. The incubation period of coronavirus disease 2019 (COVID-19) from publicly reported confirmed cases: estimation and application. Ann Intern Med. 2020. https://doi.org/10.7326/M20-0504.

8. Guan W-J, Ni Z-Y, Hu Y, et al. Clinical characteristics of coronavirus disease 2019 in China. N Engl J Med. 2020. https://doi.org/10. 1056/NEJMoa2002032.

9. Wilson N, Kvalsvig A, Barnard LT, Baker MG. Case-fatality risk estimates for COVID-19 calculated by using a lag time for fatality. Emerg Infect Dis. 2020. https://doi.org/10.3201/eid2606.200320.

10. Cai J, Xu J, Lin D, et al. A case series of children with 2019 novel coronavirus infection: clinical and epidemiological features. Clin Infect Dis. 2020. https://doi.org/10.1093/cid/ciaa198.

11. Ai T, Yang Z, Hou H, et al. Correlation of chest CT and RT-PCR testing in coronavirus disease 2019 (COVID-19) in China: a report of 1014 cases. Radiology. 2020. https://doi.org/10.1148/radiol. 2020200642

12. Zimmermann P, Curtis N. Coronavirus infections in children including COVID-19: an overview of the epidemiology, clinical features, diagnosis, treatment and prevention options in children. Pediatr Infect Dis J. 2020. https://doi.org/10.1097/INF. 0000000000002660 .

13. Revised Strategy of COVID19 testing in India (Version 3, dated 20/ 03/2020). Available at: https://icmr.nic.in/sites/default/files/upload documents/2020-03-20_covid19_test_v3.pdf. Accessed 31 March 2020.

14. Xia W, Shao J, Guo Y, Peng X, Li Z, Hu D. Clinical and CT features in pediatric patients with COVID-19 infection: different points from adults. Pediatr Pulmonol. 2020. https://doi.org/10.1002/ppul. 24718.

15. Lu X, Zhang L, Du H, et al. SARS-CoV-2 infection in children. N Engl J Med. 2020. https://doi.org/10.1056/NEJMc2005073.

16. Dong Y, Mo X, Hu Y, et al. Epidemiological characteristics of 2143 pediatric patients with 2019 coronavirus disease in China. Pediatrics. 2020. https://doi.org/10.1542/peds.2020-0702.

17. Chen Z-M, Fu J-F, Shu Q, et al. Diagnosis and treatment recommendations for pediatric respiratory infection caused by the 2019 novel coronavirus. World J Pediatr. 2020. https://doi.org/10.1007/ s12519-020-00345-5.

18. Wu Z, McGoogan JM. Characteristics of and important lessons from the coronavirus disease 2019 (COVID-19) outbreak in China: summary of a report of 72314 cases from the Chinese Center for Disease Control and Prevention. JAMA. 2020. https:// doi.org/10.1001/jama.2020.2648.

19. Guidelines for use of commercial kits for nasal/throat swab based diagnosis of COVID-19 in India, 24 March, 2020. Available at: https://icmr.nic.in/sites/default/files/upload_documents/Rev Guidelines_for_use_of_commercial_kits_for_diagnosis_of_ COVID 240320.pdf. Accessed 31 March 2020.

20. ICMR. Advisory to start rapid antibody based blood test for COVID-19 (4 April 2020). Available at: https://icmr.nic.in/sites/ default/files/upload_documents/Advisory_Antibody_Testing 04042020.pdf. Accessed 5 April 2020.

21. Clinical management of severe acute respiratory infection when COVID-19 is suspected. Available at: https://www.who.int/ publications-detail/clinical-management-of-severe-acuterespiratory-infection-when-novel-coronavirus-(ncov)-infection-issuspected. Accessed 31 March 2020.

22. Infection prevention \& control guidelines for 2019-nCoV (COVID19). Available at: https://www.aiims.edu/images/pdf/notice/ Updated_COVID_19_HIC_SUPPLEMENT_VERSION_1.2 30_March_2020.pdf. Accessed 31 March 2020.

23. Wong HYF, Lam HYS, Fong AH, et al. Frequency and distribution of chest radiographic findings in COVID-19 positive patients. Radiology. 2020. https://doi.org/10.1148/radiol.2020201160.

24. Simonds AK, Hanak A, Chatwin M, et al. Evaluation of droplet dispersion during non-invasive ventilation, oxygen therapy, nebuliser treatment and chest physiotherapy in clinical practice: implications for management of pandemic influenza and other airborne infections. Health Technol Assess. 2010;14:131-72.

25. Liu J, Cao R, Xu M, et al. Hydroxychloroquine, a less toxic derivative of chloroquine, is effective in inhibiting SARS-CoV-2 infection in vitro. Cell Discov. 2020;6:1-4.

26. Gautret P, Lagier J-C, Parola P, et al. Hydroxychloroquine and azithromycin as a treatment of COVID-19: results of an openlabel non-randomized clinical trial. Int J Antimicrob Agents. 2020. https://doi.org/10.1016/j.ijantimicag.2020.105949.

27. Cao B, Wang $\mathrm{Y}$, Wen $\mathrm{D}$, et al. A trial of lopinavir-ritonavir in adults hospitalized with severe Covid-19. N Engl J Med. 2020. https://doi. org/10.1056/NEJMoa2001282.

28. Morse JS, Lalonde T, Xu S, Liu WR. Learning from the past: possible urgent prevention and treatment options for severe acute respiratory infections caused by $2019-\mathrm{nCoV}$. Chembiochem. $2020 ; 21: 730-8$. 
29. Russell B, Moss C, George G, et al. Associations between immunesuppressive and stimulating drugs and novel COVID-19-a systematic review of current evidence. Ecancer. 2020. https://doi.org/ 10.3332/ecancer.2020.1022.

30. Shen C, Wang Z, Zhao F, et al. Treatment of 5 critically ill patients with COVID-19 with convalescent plasma. JAMA. 2020. https:// doi.org/10.1001/jama.2020.4783.

31. Pediatric Acute Lung Injury Consensus Conference Group. Pediatric acute respiratory distress syndrome: consensus recommendations from the pediatric acute lung injury consensus conference. Pediatr Crit Care Med. 2015;16:428-39.
32. Kabra SK, Lodha R. Pediatric Intensive Care Protocols of AIIMS, 2nd ed. India: Indian J Pediatr; 2017.

33. Weiss SL, Peters MJ, Alhazzani W, et al. Surviving Sepsis campaign international guidelines for the management of septic shock and sepsis-associated organ dysfunction in children. Pediatr Crit Care Med. 2020;21:e52-106.

Publisher's Note Springer Nature remains neutral with regard to jurisdictional claims in published maps and institutional affiliations. 